TRANSACTIONS OF THE

AMERICAN MATHEMATICAL SOCIETY

Volume 348, Number 1, January 1996

\title{
COMPLEX CONVEXITY IN LEBESGUE-BOCHNER FUNCTION SPACES
}

\author{
PATRICK N. DOWLING, ZHIBAO HU, AND DOUGLAS MUPASIRI
}

\begin{abstract}
Complex geometric properties of continuously quasi-normed spaces are introduced and their relationship to their analogues in real Banach spaces is discussed. It is shown that these properties lift from a continuously quasi-normed space $X$ to $L^{p}(\mu, X)$, for $0<p<\infty$. Local versions of these properties and results are also considered.
\end{abstract}

\section{INTRODUCTION}

There are many papers in the literature dealing with the problem of lifting a property of a Banach space $X$ to the Lebesgue-Bochner function spaces $L^{p}(\mu, X)$, for $1<p<\infty$. In particular, several of these articles deal with lifting a geometric property of $X$ to $L^{p}(\mu, X)$ (see the survey paper of Smith [10] and the references therein). Specifically, it is now well-known that strict convexity, uniform convexity, local uniform convexity, and midpoint local uniform convexity all lift from $X$ to $L^{p}(\mu, X)$ when $1<p<\infty$. In the late 1960's and early 1970's Sundaresan [12, 13] and Johnson [7] considered local versions of these results. One such result was that of Johnson [7], where it was shown that if $X$ is a separable Banach space and $f$ is a norm 1 element of $L^{p}(\mu, X)$, for $1<p<\infty$, then $f$ is an extreme point of the unit ball of $L^{p}(\mu, X)$ if and only if $f(\omega) /\|f(\omega)\|$ is an extreme point of $B_{X}$ for $\mu$-almost all $\omega$ in the support of $f$. Later, Greim [4] showed that the separability condition on $X$ was essential. Results of a similar ilk can be found in $[5,9,10]$.

In the early 1980's, much work in the area of the geometry of Banach spaces was directed at the complex geometry of complex Banach spaces. Davis, Garling, and Tomczak-Jaegermann [1] introduced the notion of uniform PL-convexity as a complex analogue of uniform convexity in real Banach spaces. Uniform PLconvex spaces exhibit some of the nice features enjoyed by uniform convex spaces, but it is unknown whether a Banach space which is uniformly PL-convex can be renormed to be uniformly PL-convex, where the modulus of uniform PL-convexity is of power type [1]. Xu [15] offered his own complex analogue of uniform convexity called uniform H-convexity. He showed that if a Banach space is uniformly $\mathrm{H}$ convex, then it can be equivalently renormed to be uniformly H-convex where the modulus of uniform H-convexity is of power type [15]. Both of these "complex uniform convexity" properties are not measures of convexity but rather measures of subharmonicity and so it was possible to define these properties not only for Banach

Received by the editors July 22, 1994.

1991 Mathematics Subject Classification. Primary 28A05, 46E40.

Key words and phrases. Quasi-normed spaces, complex extreme points, complex strongly extreme points, analytic denting points.

(C)1996 American Mathematical Society 
spaces but for continuously quasi-normed spaces (see section 2). The complex analogue of strict convexity is complex strict convexity. Complex strict convexity was introduced by Thorp and Whitley [14] for complex Banach spaces, and is a measure of convexity. However, it was shown by Dilworth [2], that it can be viewed as a measure of subharmonicity.

There are some lifting results known for these complex geometric properties. For example, if $X$ is a continuously quasi-normed space which is uniformly PLconvex (respectively, uniformly H-convex), then $L^{p}(\mu, X)$ is uniformly PL-convex (respectively, uniformly $\mathrm{H}$-convex), for $0<p<\infty$ [1] (respectively, [14]). If $X$ is a Banach space which is complex strictly convex, then $L^{p}(\mu, X)$ is complex strictly convex for $0<p<\infty[2]$. This last result is somewhat unsatisfactory because the requirement that $X$ be a Banach space is too restrictive. In particular, the result provides no information about the complex strict convexity of $L^{p}\left(\mu, L^{q}(\mu)\right)$ when $0<p, q<1$, even though $L^{p}(\mu)$ and $L^{q}(\mu)$ are both complex strictly convex. The result has not been improved because of the lack of a suitable definition of complex strict convexity in continuously quasi-normed spaces. We will remedy the situation in this paper.

In section 2, we introduce the notion of complex strict convexity of a continuously quasi-normed space and more generally introduce the notion of a complex extreme point of the unit ball of a continuously quasi-normed space, which is the complex analogue of an extreme point of the unit ball of a Banach space. We also introduce the notion of complex strongly extreme point and analytic denting point which are complex analogues of strongly extreme point and denting point, respectively. Analytic denting points were motivated by the characterization of denting points in [3]. In section 3, we prove lifting results for these properties from a continuously quasi-normed space $X$ to $L^{p}(\mu, X)$ where $0<p<\infty$. The statements of these results are similar to their real counterparts $[5,7,9,10 \& 11]$ but the proofs are quite different, and in fact, the proofs can be modified to recover their corresponding real analogues.

\section{Preliminaries AND DEFinitions}

A quasi-normed space is a pair $(X,\|\cdot\|)$ where $X$ is a vector space and $\|\cdot\|$ is a function on $X$ with values in $\mathbb{R}^{+}$satisfying

(a) $\|\lambda x\|=|\lambda|\|x\|$ for all scalars $\lambda$ and all $x$ in $X$;

(b) there exists $C>0$ such that $\|x+y\| \leq C[\|x\|+\|y\|]$ for all $x$ and $y$ in $X$; and,

(c) $\|x\|=0$ if and only if $x=0$.

The smallest $C$ for which (b) holds is called the quasi-norm constant of $(X,\|\cdot\|)$.

A quasi-normed space $(X,\|\cdot\|)$ is said to be a continuously quasi-normed space if $\|\cdot\|$ is uniformly continuous on the bounded subsets of $X$. We shall call a complete continuously quasi-normed space a continuous quasi-Banach space. A continuous quasi-Banach space $(X,\|\cdot\|)$ is said to be locally PL-convex if $\log \|\cdot\|$ is a plurisubharmonic function on $X[1$, Proposition 2.2].

If $X$ is a continuous quasi-Banach space, then $B_{X}$ will denote the unit ball of $X$ and $S_{X}$ will denote the unit sphere of $X$.

Throughout this paper, $D$ will denote the open unit disc in the complex plane, and $\mathbb{T}$ will denote its boundary. 
Definition 1. Let $X$ be a complex Banach space and let $x \in S_{X}$. Then $x$ is called a complex extreme point of $B_{X}$ if for every non-zero $y \in X$, there exists $z \in D$ so that $\|x+z y\|>1$.

A Banach space is said to be complex strictly convex if every element of $S_{X}$ is a complex extreme point of $B_{X}$.

The following characterization of complex extreme points can be found in [8].

Theorem 2. Let $X$ be a complex Banach space and let $x \in S_{X}$. Then the following conditions are equivalent:

(i) $x$ is a complex extreme point of $B_{X}$;

(ii) there exists $0<p<\infty$ such that for all non-zero $y \in X, \int_{0}^{2 \pi}\left\|x+e^{i \theta} y\right\|^{p} \frac{d \theta}{2 \pi}>$ 1 ;

(iii) for each $0<p<\infty$ and for each non-zero, $y \in X, \int_{0}^{2 \pi}\left\|x+e^{i \theta} y\right\|^{p} \frac{d \theta}{2 \pi}>1$.

From Theorem 2, we are naturally led to the following:

Definition 3. Let $X$ be a continuous quasi-Banach space. A point $x \in S_{X}$ is said to be a complex extreme point of $B_{X}$ if for each non-zero $y \in X$,

$$
\int_{0}^{2 \pi}\left\|x+e^{i \theta} y\right\| \frac{d \theta}{2 \pi}>1
$$

We say that $X$ is complex strictly convex if every element of $S_{X}$ is a complex extreme point of $B_{X}$.

Remarks.

1. Clearly Definitions 1 and 3 are equivalent in Banach spaces because of Theorem 2.

2. Let $X$ be a locally PL-convex continuous quasi-Banach space. A point $x \in S_{X}$ is a complex extreme point of $B_{X}$ if and only if for some $0<p<\infty$ (or for all $0<p<\infty)$ and all non-zero $y \in X, \int_{0}^{2 \pi}\left\|x+e^{i \theta} y\right\|^{p} \frac{d \theta}{2 \pi}>1$. This follows from [1,Theorem 2.4].

3. Note that to obtain the result in Remark 2 one can relax the condition that $X$ is locally PL-convex, by only insisting that the function $\log \|x+z y\|$ is a subharmonic in the complex variable $z$ for each $y \in X$. However, in most applications one deals with locally PL-convex continuous quasi-Banach spaces, so from now on we will restrict our attention to that class of spaces.

A strengthening of the notion of complex extreme point is the following:

Definition 4. Let $X$ be a locally PL-convex continuous quasi-Banach space. A point $x \in S_{X}$ is said to be a complex strongly extreme point of $B_{X}$ if for each $\varepsilon>0$, there is $\delta>0$ so that $\int_{0}^{2 \pi}\left\|x+e^{i \theta} y\right\| \frac{d \theta}{2 \pi} \geq 1+\delta$, whenever $y \in X$ and $\|y\| \geq \varepsilon$.

Remarks.

1. A point $x \in S_{X}$ is a complex strongly extreme point of $B_{X}$ if and only if for some $0<p<\infty$ (or for all $0<p<\infty$ ) the following condition holds:

for each $\varepsilon>0$, there is $\delta_{p}>0$ so that $\int_{0}^{2 \pi}\left\|x+e^{i \theta} y\right\|^{p} \frac{d \theta}{2 \pi} \geq$ $1+\delta_{p}$, whenever $y \in X$ and $\|y\| \geq \varepsilon$.

This follows from [1, Theorem 2.4]. 
2. If $X$ is a complex Banach space, then a point $x \in S_{X}$ is a complex strongly extreme point of $B_{X}$ if and only if for each $\varepsilon>0$, there is $\delta>0$ such that for each $y \in X$ with $\|y\| \geq \varepsilon$, there is $z \in D$ with $\|x+z y\| \geq 1+\delta$. See [2, Proposition 2.2; 6, Theorem 7].

3. It is clear from Remark 2 that the notion of a complex strongly extreme point in a complex Banach space is the analogue of a strongly extreme point of a real Banach space.

It should be noted that complex strongly extreme points are the local analogues of uniform PL-convexity [1]. The local analogue of uniform H-convexity (see [3]) is the following:

Definition 5. Let $X$ be a locally PL-convex continuous quasi-Banach space. A point $x \in S_{X}$ is said to be an analytic denting point of $B_{X}$ if for each $\varepsilon>0$, there is $\delta>0$ so that $\int_{0}^{2 \pi}\left\|P\left(e^{i \theta}\right)\right\| \frac{d \theta}{2 \pi}>1+\delta$, where $P\left(e^{i \theta}\right)=\sum_{k=0}^{n} a_{k} e^{i k \theta}, a_{k} \in X$, for $0 \leq k \leq n$ and $n \in \mathbb{N}$, with $a_{0}=x$ and $\int_{0}^{2 \pi}\left\|P\left(e^{i \theta}\right)-x\right\| \frac{d \theta}{2 \pi} \geq \varepsilon$.

Remarks.

1. A point $x \in S_{X}$ is an analytic denting point of $B_{X}$ if and only if for some $0<p<\infty$ (or for all $0<p<\infty$ ) the following condition holds:

for each $\varepsilon>0$, there is $\delta_{p}>0$ so that $\int_{0}^{2 \pi}\left\|P\left(e^{i \theta}\right)\right\|^{p} \frac{d \theta}{2 \pi}>1+$ $\delta_{p}$, whenever $P\left(e^{i \theta}\right)=\sum_{k=0}^{n} a_{k} e^{i k \theta}, a_{k} \in X$, for $0 \leq k \leq n$ and $n \in \mathbb{N}$, with $a_{0}=x$ and $\left(\int_{0}^{2 \pi}\left\|P\left(e^{i \theta}\right)-x\right\|^{p} \frac{d \theta}{2 \pi}\right)^{\frac{1}{p}} \geq \varepsilon$.

This follows by modifying a result of [16, Theorem 2.1].

2. For $0<p<\infty, H^{p}(\mathbb{T}, X)=\left\{f \in L^{p}(\mathbb{T}, X): \hat{f}(n)=0\right.$ for all $\left.n<0\right\}$ and the norm of $H^{p}(\mathbb{T}, X)$ is the usual $L^{p}(\mathbb{T}, X)$ norm; $\|f\|_{p}=\left(\int_{0}^{2 \pi}\left\|f\left(e^{i \theta}\right)\right\|^{p} \frac{d \theta}{2 \pi}\right)^{\frac{1}{p}}$. Polynomials of the form $P\left(e^{i \theta}\right)=\sum_{k=0}^{n} a_{k} e^{i k \theta}, a_{k} \in X$, for $0 \leq k \leq n$ and $n \in \mathbb{N}$, are dense in $H^{p}(\mathbb{T}, X)$. Thus we have the following equivalent formulation of an analytic denting point:

$x \in S_{X}$ is an analytic denting point of $B_{X}$ if and only if for each $\varepsilon>0$, there is $\delta>0$ so that $\|f\|_{1} \geq 1+\delta$ whenever $f \in H^{1}(\mathbb{T}, X)$ with $\hat{f}(0)=x$ and $\|f-\hat{f}(0)\|_{1} \geq \varepsilon$.

A similar characterization of an analytic denting point can also be obtained in terms of $H^{p}(\mathbb{T}, X)$-functions using Remark 1.

3. It is clear from the definitions that analytic denting points are complex strongly extreme points. The converse is not true as the following example shows.

Example 6. The point $x=(1,1,1, \ldots)$ is a complex strongly extreme point of $B_{\ell^{\infty}}$, since it is a strongly extreme point of $B_{\ell^{\infty}}$.

On the other hand, if $a \in D$, let $\varphi_{a}$ be the Möbius transformation $\varphi_{a}(z)=\frac{z+a}{1+\bar{a} z}$. Then $\varphi_{a}$ maps $D$ onto $D$ and $\mathbb{T}$ onto $\mathbb{T}$. Also,

$$
\sup _{\theta \in \mathbb{R}}\left|\varphi_{a}\left(e^{i \theta}\right)-a\right|=1+|a| \geq 1
$$

That is, $\sup _{\theta \in \mathbb{R}}\left|\varphi_{a}\left(e^{i \theta}\right)-\hat{\varphi}_{a}(0)\right| \geq 1$. 
Let $\left\{a_{m}\right\}_{m=1}^{\infty}$ be dense in $\mathbb{T}$. Let $\left\{r_{n}\right\}_{n=1}^{\infty}$ be a sequence in $(0,1)$ with $\lim _{n \rightarrow \infty} r_{n}=1$. Define

$$
\begin{gathered}
f_{n}: \bar{D} \rightarrow \ell_{\infty} \quad \text { by } \\
f_{n}(z)=\left(\frac{1}{r_{n} a_{m}} \varphi_{r_{n} a_{m}}(z)\right)_{m=1}^{\infty} .
\end{gathered}
$$

Note that for each $z \in \mathbb{T},\left\|f_{n}(z)\right\|_{\ell^{\infty}}=\frac{1}{r_{n}}, \hat{f}_{n}(0)=(1,1,1, \ldots)$ and $f_{n}$ is analytic on $D$.

Hence $f_{n} \in H^{1}\left(\mathbb{T}, \ell^{\infty}\right)$ and $\left\|f_{n}\right\|_{1}=\frac{1}{r_{n}}$, so $\lim _{n \rightarrow \infty}\left\|f_{n}\right\|_{1}=1$. However

$$
\begin{aligned}
\left\|f_{n}-\hat{f}_{n}(0)\right\|_{1} & =\int_{0}^{2 \pi} \sup _{m \in \mathbb{N}}\left|\frac{1}{r_{n} a_{m}} \varphi_{r_{n} a_{m}}\left(e^{i \theta}\right)-1\right| \frac{d \theta}{2 \pi} \\
& =\int_{0}^{2 \pi} \frac{1}{r_{n}} \sup _{m \in \mathbb{N}}\left|\varphi_{r_{n} a_{m}}\left(e^{i \theta}\right)-\hat{\varphi}_{r_{n} a_{m}}(0)\right| \frac{d \theta}{2 \pi} \\
& \geq \frac{1}{r_{n}} \geq 1, \quad \text { for all } n \in \mathbb{N} .
\end{aligned}
$$

Thus $x$ is not an analytic denting point of $B_{X}$.

\section{Lifting THEOREMS}

Throughout this section $(\Omega, \Sigma, \mu)$ will denote a positive, complete measure space. If $X$ is a continuous quasi-Banach space and $0<p<\infty$, then $L^{p}(\mu, X)$ is the Lebesgue-Bochner space of all (equivalence classes of) strongly measurable functions $f: \Omega \rightarrow X$ for which $\|f\|_{L_{p}(\mu, X)}<\infty$, where

$$
\|f\|_{L^{p}(\mu, X)}=\left(\int_{\Omega}\|f(\omega)\|^{p} d \mu(\omega)\right)^{\frac{1}{p}} .
$$

$L^{p}(\mu)$ denotes $L^{p}(\mu, X)$, when $X$ is the scalar field.

Theorem 7. Let $X$ be a locally $P L$-convex continuous quasi-Banach space, let $0<$ $p<\infty$, and let $f \in S_{L^{p}(\mu, X)}$. Then $f$ is a complex strongly extreme (respectively, analytic denting) point of $B_{L^{p}(\mu, X)}$ if and only if $\frac{f(\omega)}{\|f(\omega)\|}$ is a complex strongly extreme (respectively, analytic denting) point of $B_{X}$, for $\mu$-almost all $\omega \in \operatorname{supp} f=$ $\{\omega \in \Omega: f(\omega) \neq 0\}$.

Proof. We will prove the theorem for analytic denting points; the result for complex strongly extreme points follows in a similar manner.

Suppose that for $\mu$-almost all $\omega \in \operatorname{supp} f, f(\omega) /\|f(\omega)\|$ is an analytic 
denting point of $B_{X}$. Let $\left\{G_{n}\right\}_{n=1}^{\infty}$ be a sequence in $H^{p}\left(\mathbb{T}, L^{p}(\mu, X)\right)$ with $\lim _{n \rightarrow \infty}\left\|G_{n}+f\right\|_{p}=1$, and $\widehat{G_{n}}(0)=0$. To show that $f$ is an analytic denting point of $B_{L^{p}(\mu, X)}$ it suffices to show that $\lim _{n \rightarrow \infty}\left\|G_{n}\right\|_{p}=0$. Since $\widehat{G_{n}}(0)=$ $0,\left(\widehat{G_{n}}(0)\right)(\omega)=0$ for $\mu$-almost all $\omega \in \Omega$. Hence

$$
\begin{aligned}
\left\|G_{n}(\cdot)+f\right\|_{p}^{p} & =\int_{0}^{2 \pi}\left\|G_{n}\left(e^{i \theta}\right)+f\right\|_{L^{p}(\mu, X)}^{p} \frac{d \theta}{2 \pi} \\
& =\int_{0}^{2 \pi} \int_{\Omega}\left\|G_{n}\left(e^{i \theta}\right)(\omega)+f(\omega)\right\|^{p} d \mu(\omega) \frac{d \theta}{2 \pi} \\
& =\int_{\Omega} \int_{0}^{2 \pi}\left\|G_{n}\left(e^{i \theta}\right)(\omega)+f(\omega)\right\|^{p} \frac{d \theta}{2 \pi} d \mu(\omega) .
\end{aligned}
$$

Define $g_{n}(\omega)=\left(\int_{0}^{2 \pi}\left\|G_{n}\left(e^{i \theta}\right)(\omega)+f(\omega)\right\|^{p} \frac{d \theta}{2 \pi}\right)^{\frac{1}{p}}$.

Note that $g_{n}(\omega) \geq\|f(\omega)\|$ for $\mu$-almost all $\omega \in \Omega$ and

$$
\begin{aligned}
\left\|G_{n}(\cdot)+f\right\|_{p}^{p} & =\int_{\Omega} g_{n}(\omega)^{p} d \mu(\omega) \\
& \rightarrow 1=\int_{\Omega}\|f(\omega)\|^{p} d \mu(\omega) .
\end{aligned}
$$

Hence, there is a subsequence $\left\{g_{n_{k}}\right\}_{k=1}^{\infty}$ of $\left\{g_{n}\right\}_{n=1}^{\infty}$ converging pointwise $\mu$-almost everywhere to $\|f\|$.

Note that

$$
\begin{aligned}
\left\|G_{n_{k}}\right\|_{p}^{p} & =\int_{0}^{2 \pi}\left\|G_{n_{k}}\left(e^{i \theta}\right)\right\|_{L^{p}(\mu, X)}^{p} \frac{d \theta}{2 \pi} \\
& =\int_{0}^{2 \pi} \int_{\Omega}\left\|G_{n_{k}}\left(e^{i \theta}\right)(\omega)\right\|^{p} d \mu(\omega) \frac{d \theta}{2 \pi} \\
& =\int_{\Omega} \int_{0}^{2 \pi}\left\|G_{n_{k}}\left(e^{i \theta}\right)(\omega)\right\|^{p} \frac{d \theta}{2 \pi} d \mu(\omega) \\
& =\int_{\Omega}\left\|G_{n_{k}}(\cdot)(\omega)\right\|_{p}^{p} d \mu(\omega) .
\end{aligned}
$$

Also, since $\left\|G_{n_{k}}(\cdot)(\omega)+f(\omega)\right\|_{p}=g_{n_{k}}(\omega) \stackrel{k}{\rightarrow}\|f(\omega)\|$ and $\widehat{G_{n_{k}}}(0)(\omega)=0$ for $\mu$ almost all $\omega \in \Omega,\left\|G_{n_{k}}(\cdot)(\omega)\right\|_{p} \longrightarrow 0$ for $\mu$-almost all $\omega \in \Omega$, because $f(\omega) /\|f(\omega)\|$ is an analytic denting point of $B_{X}^{n}$ for $\mu$-almost all $\omega \in \operatorname{supp} f$. 


$$
\begin{aligned}
\left\|G_{n_{k}}(\cdot)(\omega)\right\|_{p}^{p} & =\int_{0}^{2 \pi}\left\|G_{n_{k}}\left(e^{i \theta}\right)(\omega)\right\|^{p} \frac{d \theta}{2 \pi} \\
& =\int_{0}^{2 \pi}\left\|G_{n_{k}}\left(e^{i \theta}\right)(\omega)+f(\omega)-f(\omega)\right\|^{p} \frac{d \theta}{2 \pi} \\
& \leq \int_{0}^{2 \pi}\left\{C\left[\left\|G_{n_{k}}\left(e^{i \theta}\right)(\omega)+f(\omega)\right\|+\|f(\omega)\|\right]\right\}^{p} \frac{d \theta}{2 \pi}
\end{aligned}
$$

(where $C$ is the quasi-norm constant of $X$ )

$$
\begin{aligned}
& \leq \int_{0}^{2 \pi}(2 C)^{p}\left[\left\|G_{n_{k}}\left(e^{i \theta}\right)(\omega)+f(\omega)\right\|^{p}+\|f(\omega)\|^{p}\right] \frac{d \theta}{2 \pi} \\
& =(2 C)^{p}\left[g_{n_{k}}(\omega)^{p}+\|f(\omega)\|^{p}\right] .
\end{aligned}
$$

Since $\left\{(2 C)^{p}\left[g_{n_{k}}(\cdot)^{p}+\|f(\cdot)\|^{p}\right]\right\}_{k=1}^{\infty}$ converges in $L^{1}(\mu)$ and since $\left\|G_{n_{k}}(\cdot)(\omega)\right\|_{p}^{p} \underset{k}{\longrightarrow}$ 0 , for $\mu$-almost all $\omega \in \Omega$, we have by a variation of the Lebesgue Dominated Convergence Theorem that

$$
\left\|G_{n_{k}}\right\|_{p}^{p}=\int_{\Omega}\left\|G_{n_{k}}(\cdot)(\omega)\right\|_{p}^{p} d \mu(\omega) \underset{k}{\longrightarrow} 0
$$

By repeating this argument for each subsequence of $\left\{G_{n_{k}}\right\}_{n=1}^{\infty}$ we conclude that $\left\|G_{n}\right\|_{p} \underset{n}{\longrightarrow} 0$. Therefore, $f$ is an analytic denting point of $B_{L^{p}(\mu, X)}$.

Conversely, suppose that

$$
E=\left\{\omega \in \operatorname{supp} f: f(\omega) /\|f(\omega)\| \text { is not an analytic denting point of } B_{X}\right\}
$$

is not a $\mu$-null set. Then $\mu^{*}(E)>0$, where $\mu^{*}$ is the outer measure of $\mu$. Define

$$
\begin{aligned}
E_{n}= & \left\{\omega \in \Omega: \text { there exists }\left\{g_{k}\right\}_{k=1}^{\infty} \text { in } H^{p}(\mathbb{T}, X), \text { with } \hat{g}_{k}(0)=0,\right. \\
& \left.\left\|g_{k}+f(\omega)\right\|_{p} \leq\|f(\omega)\|+\frac{1}{k},\left\|g_{k}\right\|_{p} \geq \frac{1}{n}, \text { for all } k \in \mathbb{N}, \text { and }\|f(\omega)\| \leq n\right\}
\end{aligned}
$$

Clearly, $E=\bigcup_{n=1}^{\infty} E_{n}$ and so there is $n_{0} \in \mathbb{N}$ such that $\mu^{*}\left(E_{n_{0}}\right)>0$. Without loss of generality we can also assume that $\mu^{*}\left(E_{n_{0}}\right)<\infty$. Therefore, there exists $F \in \Sigma$ such that $E_{n_{0}} \subset F, \mu(F)=\mu^{*}\left(E_{n_{0}}\right)$ and $\|f(\omega)\| \leq n_{0}$ for all $\omega \in F$. Let $\varepsilon_{0}=\mu^{*}\left(E_{n_{0}}\right) / 2$.

Let $C$ be the quasi-norm constant of $L^{p}(\mathbb{T}, X)$. Choose $M>0$ so that $(1+x)^{\frac{1}{p}} \leq$ $1+M x^{\min \left\{\frac{1}{p}, 1\right\}}$ for all $x \in\left[0, \varepsilon_{0}\right]$.

If $\delta>0$ is given, choose $0<\varepsilon_{1}<\frac{1}{6}$ so that $M\left(3 \varepsilon_{1} \mu^{*}\left(E_{n_{0}}\right)\right)^{\min \left\{\frac{1}{p}, 1\right\}}<\delta$. Since $X$ is a continuous quasi-Banach space, so is $L^{p}(\mathbb{T}, X)$ [1, Proposition 2.1], and hence $\|\cdot\|^{p}$ and $\|\cdot\|_{L^{p}(\mathbb{T}, X)}^{p}$ are uniformly continuous functions on bounded subsets of $X$ and $L^{p}(\mathbb{T}, X)$ respectively. Therefore there is $\delta_{1}>0$ so that $\|x+y\|^{p} \leq\|x\|^{p}+\varepsilon_{1}$ 
whenever $\|x\| \leq 2 n_{0} C$ and $\|y\|<\delta_{1}$, and $\|h+k\|_{L^{p}(\mathbb{T}, X)}^{p} \leq\|h\|_{L^{p}(\mathbb{T}, X)}^{p}+\varepsilon_{1}$ whenever $\|h\|_{L^{p}(\mathbb{T}, X)} \leq n_{0}+1$ and $\|k\|_{L^{p}(\mathbb{T}, X)}<\delta_{1}$.

Since $f$ is measurable and $\mu(F)>\varepsilon_{0}$, there exists pairwise disjoint, measurable subsets $F_{1}, \ldots, F_{k}$ of $F$ each of positive $\mu$-measure and $\sum_{j=1}^{k} \mu\left(F_{j}\right)>\varepsilon_{0}$ and $\operatorname{diam} f\left(F_{j}\right)<\delta$, for each $j=1,2, \ldots, k$. Clearly, $\mu^{*}\left(F_{j} \cap E\right)=\mu\left(F_{j}\right)>0$ for each $j=1, \ldots, k$. Choose $\omega_{j} \in F_{j} \cap E$ for each $j=1, \ldots, k$. Choose $g_{j} \in H^{p}(\mathbb{T}, X)$ with $\hat{g}_{j}(0)=0,\left\|g_{j}\right\|_{p}>\frac{1}{n_{0}}$, and $\left\|g_{j}+f\left(\omega_{j}\right)\right\|_{p}^{p}<\left\|f\left(\omega_{j}\right)\right\|^{p}+\varepsilon_{1}$. Define

$$
G: \mathbb{T} \rightarrow L^{p}(\mu, X)
$$

by

$$
G\left(e^{i \theta}\right)=\sum_{j=1}^{k} g_{j}\left(e^{i \theta}\right) \chi_{F_{j}}
$$

Clearly, $G \in H^{p}\left(\mathbb{T}, L^{p}(\mu, X)\right), \hat{G}(0)=0$, and

$$
\begin{aligned}
\|G\|_{p} & =\left(\int_{0}^{2 \pi}\left\|G\left(e^{i \theta}\right)\right\|_{L^{p}(\mu, X)}^{p} \frac{d \theta}{2 \pi}\right)^{\frac{1}{p}} \\
& =\left[\int_{0}^{2 \pi} \int_{\Omega}\left\|G\left(e^{i \theta}\right)(\omega)\right\|^{p} d \mu(\omega) \frac{d \theta}{2 \pi}\right]^{\frac{1}{p}} \\
& =\left[\int_{\Omega} \int_{0}^{2 \pi}\left\|G\left(e^{i \theta}\right)(\omega)\right\|^{p} \frac{d \theta}{2 \pi} d \mu(\omega)\right]^{\frac{1}{p}} \\
& =\left[\int_{\Omega} \int_{0}^{2 \pi}\left(\sum_{j=1}^{k}\left\|g_{j}\left(e^{i \theta}\right)\right\|^{p} \chi_{F_{j}}(\omega)\right)^{\frac{d \theta}{2 \pi}} d \mu(\omega)\right]^{\frac{1}{p}} \\
& =\left[\int_{\Omega}\left(\sum_{j=1}^{k}\left\|g_{j}\right\|_{p}^{p} \chi_{F_{j}}(\omega)\right) d \mu(\omega)\right]^{\frac{1}{p}} \\
\geq & \frac{1}{n_{0}} \mu\left(\bigcup_{j=1}^{k} F_{j}\right)^{\frac{1}{p}} \geq \frac{1}{n_{0}} \varepsilon_{0}^{\frac{1}{p}}=\frac{\varepsilon_{0}^{\frac{1}{p}}}{n_{0}} .
\end{aligned}
$$

On the other hand, for all $\omega \in \Omega$

$$
\left\|G\left(e^{i \theta}\right)(\omega)+f(\omega)\right\|^{p}=\left\{\begin{array}{l}
\|f(\omega)\|^{p} ; \text { if } \omega \in \Omega \backslash \bigcup_{j=1}^{k} F_{j}, \\
\left\|g_{j}\left(e^{i \theta}\right)+f(\omega)\right\|^{p} ; \text { if } \omega \in F_{j} .
\end{array}\right.
$$


Hence, if $\omega \in \Omega \backslash \bigcup_{j=1}^{k} F_{j}$,

$$
\int_{0}^{2 \pi}\left\|G\left(e^{i \theta}\right)(\omega)+f(\omega)\right\|^{p} \frac{d \theta}{2 \pi}=\|f(\omega)\|^{p},
$$

and if $\omega \in F_{j}$, then

$$
\begin{aligned}
\int_{0}^{2 \pi}\left\|G\left(e^{i \theta}\right)(\omega)+f(\omega)\right\|^{p} \frac{d \theta}{2 \pi} & =\int_{0}^{2 \pi}\left\|g_{j}\left(e^{i \theta}\right)+f(\omega)\right\|^{p} \frac{d \theta}{2 \pi} \\
& =\left\|g_{j}+f(\omega)\right\|_{L^{p}(\mathbb{T}, X)}^{p} \\
& =\left\|g_{j}+f\left(\omega_{j}\right)+\left(f(\omega)-f\left(\omega_{j}\right)\right)\right\|_{L^{p}(\mathbb{T}, X)}^{p} \\
& \leq\left\|g_{j}+f\left(\omega_{j}\right)\right\|_{L^{p}(\mathbb{T}, X)}^{p}+\varepsilon_{1} \\
& \leq\left\|f\left(\omega_{j}\right)\right\|^{p}+2 \varepsilon_{1} \\
& =\left\|f(\omega)+f\left(\omega_{j}\right)-f(\omega)\right\|^{p}+2 \varepsilon_{1} \\
& \leq\|f(\omega)\|^{p}+3 \varepsilon_{1},
\end{aligned}
$$

where the first inequality follows from the fact that $\left\|g_{j}+f\left(\omega_{j}\right)\right\|_{L^{p}(\mathbb{T}, X)} \leq n_{0}+1$ and $\left\|f(\omega)-f\left(\omega_{j}\right)\right\|_{L^{p}(\mathbb{T}, X)}<\delta_{1}$, and the last inequality follows because $\|f(\omega)\| \leq 2 n_{0} C$ and $\left\|f(\omega)-f\left(\omega_{j}\right)\right\|<\delta_{1}$.

Therefore,

$$
\int_{0}^{2 \pi}\left\|G\left(e^{i \theta}\right)(\omega)+f(\omega)\right\|^{p} \frac{d \theta}{2 \pi} \leq\|f(\omega)\|^{p}+3 \varepsilon_{1} \chi_{\cup_{j=1}^{k} F_{j}}(\omega) .
$$

Consequently,

$$
\begin{aligned}
\|G(\cdot)+f\|_{p} & =\left[\int_{0}^{2 \pi} \int_{\Omega}\left\|G\left(e^{i \theta}\right)(\omega)+f(\omega)\right\|^{p} d \mu(\omega) \frac{d \theta}{2 \pi}\right]^{\frac{1}{p}} \\
& =\left[\int_{\Omega} \int_{0}^{2 \pi}\left\|G\left(e^{i \theta}\right)(\omega)+f(\omega)\right\|^{p} \frac{d \theta}{2 \pi} d \mu(\omega)\right]^{\frac{1}{p}} \\
& \leq\left[\int_{\Omega}\|f(\omega)\|^{p}+3 \varepsilon_{1} \chi_{k}(\omega) d \mu(\omega)\right]_{j=1}^{\frac{1}{p}} F_{j} \\
& =\left[\|f\|^{p}+3 \varepsilon_{1} \mu\left(\bigcup_{j=1}^{k} F_{j}\right)\right]^{\frac{1}{p}}
\end{aligned}
$$




$$
\begin{aligned}
& \leq\|f\|+M\left(3 \varepsilon_{1} \mu\left(\bigcup_{j=1}^{k} F_{j}\right)\right)^{\min \left\{\frac{1}{p}, 1\right\}} \\
& \leq\|f\|+M\left(3 \varepsilon_{1} \mu(F)\right)^{\min \left\{\frac{1}{p}, 1\right\}} \\
& \leq\|f\|+\delta .
\end{aligned}
$$

It follows that for each $n \in \mathbb{N}$, there exists $G_{n} \in H^{p}\left(\mathbb{T}, L^{p}(\mu, X)\right)$ such that $\left\|G_{n}\right\|_{p} \geq \frac{\varepsilon_{0}^{\frac{1}{p}}}{n_{0}}, \hat{G}_{n}(0)=0$ and $\left\|G_{n}(\cdot)+f\right\|_{p}<\|f\|+\frac{1}{n}$.

Hence $f$ is not an analytic denting point of $B_{L^{p}(\mu, X)}$.

Theorem 8. Let $X$ be a locally PL-convex continuous quasi-Banach space, let $0<p<\infty$ and let $f \in S_{L^{p}(\mu, X)}$.

(a) If $f(\omega) /\|f(\omega)\|$ is a complex extreme point of $B_{X}$ for $\mu$-almost all $\omega \in \operatorname{supp} f$, then $f$ is a complex extreme point of $B_{L^{p}(\mu, X)}$.

(b) If $X$ is separable and $f$ is a complex extreme point of $B_{L^{p}(\mu, X)}$, then $f(\omega) /\|f(\omega)\|$ is a complex extreme point of $B_{X}$ for $\mu$-almost all $\omega \in \operatorname{supp} f$.

Proof. (a) Suppose that $g \in L^{p}(\mu, X)$ satisfies $\left(\int_{0}^{2 \pi}\left\|f+e^{i \theta} g\right\|_{L^{p}(\mu, X)}^{p} \frac{d \theta}{2 \pi}\right)^{\frac{1}{p}}=$ $\|f\|_{L^{p}(\mu, X)}$. Then

$$
\begin{aligned}
\left(\int_{\Omega}\|f(\omega)\|^{p} d \mu(\omega)\right)^{\frac{1}{p}} & =\left(\int_{0}^{2 \pi} \int_{\Omega}\left\|f(\omega)+e^{i \theta} g(\omega)\right\|^{p} d \mu(\omega) \frac{d \theta}{2 \pi}\right)^{\frac{1}{p}} \\
& =\left(\int_{\Omega} \int_{0}^{2 \pi}\left\|f(\omega)+e^{i \theta} g(\omega)\right\|^{p} \frac{d \theta}{2 \pi} d \mu(\omega)\right)^{\frac{1}{p}} .
\end{aligned}
$$

However, since $\|f(\omega)\|^{p} \leq \int_{0}^{2 \pi}\left\|f(\omega)+e^{i \theta} g(\omega)\right\|^{p} \frac{d \theta}{2 \pi}$, we have

$$
\|f(\omega)\|=\left(\int_{0}^{2 \pi}\left\|f(\omega)+e^{i \theta} g(\omega)\right\|^{p} \frac{d \theta}{2 \pi}\right)^{\frac{1}{p}}, \text { for } \mu \text {-almost all } \omega \in \Omega
$$

Clearly, if $\omega \in \Omega \backslash$ supp $f$ then $g(\omega)=0$, and since $f(\omega) /\|f(\omega)\|$ is a complex extreme point of $B_{X}$ for $\mu$-almost all $\omega \in \operatorname{supp} f, g(\omega)=0$ in $L^{p}(\mu, X)$ and so $f$ is a complex extreme point of $B_{L^{p}(\mu, X)}$.

(b) Suppose that $E=\{\omega \in \operatorname{supp} f: f(\omega) /\|f(\omega)\|$ is not a complex extreme point of $\left.B_{X}\right\}$ is not a $\mu$-null set. Then $\mu^{*}(E)>0$, where $\mu^{*}$ is the outer measure of $\mu$. Define

$$
\begin{aligned}
& E_{n}=\left\{\omega \in \Omega:\|f(\omega)\|<n \text { and there is } y \in X \text { with } \frac{1}{n}<\|y\|<n\right. \text {, and } \\
& \left.\qquad \int_{0}^{2 \pi}\left\|f(\omega)+e^{i \theta} y\right\|^{p} \frac{d \theta}{2 \pi}=\|f(\omega)\|^{p}\right\} .
\end{aligned}
$$

Then $E=\bigcup_{n=1}^{\infty} E_{n}$. Hence there is $n_{0} \in \mathbb{N}$ such that $\mu^{*}\left(E_{n_{0}}\right)>0$. Without loss of generality we may assume that $\mu^{*}\left(E_{n_{0}}\right)<\infty$. Therefore, there exists $F \in \Sigma$ such that $E_{n_{0}} \subset F, \mu(F)=\mu^{*}\left(E_{n_{0}}\right)$ and $\|f(\omega)\|<n_{0}$ for all $\omega \in F$. 
By the definition of $E_{n_{0}}$, there exists a function $g: E_{n_{0}} \rightarrow X$ such that $\frac{1}{n_{0}}<$ $\|g(\omega)\|<n_{0}$ and $\int_{0}^{2 \pi}\left\|f(\omega)+e^{i \theta} g(\omega)\right\|^{p} d \mu(\omega)=\|f(\omega)\|^{p}$, for all $\omega \in E_{n_{0}}$. Since $\|\cdot\|^{p}$ is uniformly continuous on bounded subsets of $X$, there is a sequence of positive real numbers $\left(\delta_{n}\right)_{n}$ with $\delta_{n} \rightarrow 0$ and satisfying $\|x\|^{p}-2^{-n} \leq\|x+y\|^{p} \leq\|x\|^{p}+2^{-n}$, for all $\|x\| \leq 2 n_{0} C$ and $\|y\| \leq \delta_{n}$. Let $\Delta=\bigcup_{n=1}^{\infty} \mathbb{N}^{n}$ and let $\Delta_{n}=\mathbb{N}^{n}$. If $\alpha=\left(i_{1}, \ldots, i_{m}\right)$ and $\beta \in\left(j_{1}, \ldots, j_{n}\right)$ are elements of $\Delta$, then we write $\alpha \leq \beta$ if $m \leq n$ and $i_{1}=j_{1}, \ldots, i_{m}=j_{m}$. Since $f$ is measurable and $X$ is separable, we can inductively construct a sequence $\left\{\Pi_{n}\right\}_{n=1}^{\infty}$ of countable partitions of $F$ with the following properties:

$\Pi_{n}=\left\{F_{\alpha}\right\}_{\alpha \in \Delta_{n}}, F_{\alpha} \supset F_{\beta}$ if $\alpha, \beta \in \Delta$ with $\alpha \leq \beta$, and there is $A_{\alpha} \subset F_{\alpha} \cap E_{n_{0}}$ such that $\mu^{*}\left(A_{\alpha}\right)=\mu\left(F_{\alpha}\right), A_{\alpha} \supset A_{\beta}$ if $\alpha \leq \beta$, and with both $\operatorname{diam} f\left(F_{\alpha}\right)<\delta_{n}$ and $\operatorname{diam} g\left(A_{\alpha}\right)<\delta_{n}$, for all $\alpha \in \Delta_{n}$.

If $\alpha \in \Delta_{n}$, and $A_{\alpha} \neq \emptyset$, choose $\omega_{\alpha} \in A_{\alpha}$. Define

$$
g_{n}=\sum_{\alpha \in \Delta_{n}} g\left(\omega_{\alpha}\right) \chi_{F_{\alpha}}
$$

Clearly $g_{n} \in L^{p}(\mu, X)$ and for all $\omega \in F, \frac{1}{n_{0}}<\left\|g_{n}(\omega)\right\|<n_{0}$. Also $\left\|g_{n}(\omega)\right\|=0$ for all $\omega \in \Omega \backslash F$. Note that for all $m \in \mathbb{N},\left\|g_{n+m}(\omega)-g_{n}(\omega)\right\| \leq \delta_{n}$ for all $\omega \in \Omega$, and since $\delta_{n} \rightarrow 0,\left\{g_{n}\right\}_{n=1}^{\infty}$ converges pointwise, to a measurable function, $h$. It is easily seen that $h \in L^{p}(\mu, X), h(\omega)=0$ for all $\omega \in \Omega \backslash F,\left\|g_{n}(\omega)-h(\omega)\right\| \leq \delta_{n}$ for all $\omega \in F$, and $\frac{1}{n_{0}} \leq\|h(\omega)\| \leq n_{0}$ for all $\omega \in F$.

If $\omega \in \Omega \backslash F$, then $\left\|f(\omega)+e^{i \theta} h(\omega)\right\|^{p}=\|f(\omega)\|^{p}$ for all $\theta \in \mathbb{R}$. If $\omega \in F$ and $n \in \mathbb{N}$, then there is a unique $\alpha \in \Delta_{n}$ so that $\omega \in F_{\alpha}$. Thus

$$
\begin{aligned}
\int_{0}^{2 \pi}\left\|f(\omega)+e^{i \theta} h(\omega)\right\|^{p} \frac{d \theta}{2 \pi} & =\int_{0}^{2 \pi}\left\|f(\omega)+e^{i \theta} g_{n}(\omega)+e^{i \theta}\left[h(\omega)-g_{n}(\omega)\right]\right\|^{p} \frac{d \theta}{2 \pi} \\
& \leq \int_{0}^{2 \pi}\left[\left\|f(\omega)+e^{i \theta} g_{n}(\omega)\right\|^{p}+2^{-n}\right] \frac{d \theta}{2 \pi} \\
& =\int_{0}^{2 \pi} \| f\left(\omega_{\alpha}\right)+e^{i \theta} g_{n}(\omega)+\left(f(\omega)-f\left(\omega_{\alpha}\right) \|^{p} \frac{d \theta}{2 \pi}+2^{-n}\right. \\
& \leq \int_{0}^{2 \pi}\left[\left\|f\left(\omega_{\alpha}\right)+e^{i \theta} g_{n}(\omega)\right\|^{p}+2^{-n}\right] \frac{d \theta}{2 \pi}+2^{-n} \\
& =\int_{0}^{2 \pi}\left\|f\left(\omega_{\alpha}\right)+e^{i \theta} g_{n}\left(\omega_{\alpha}\right)\right\|^{p} \frac{d \theta}{2 \pi}+2^{-n+1} \\
& =\left\|f\left(\omega_{\alpha}\right)\right\|^{p}+2^{-n+1} \\
& =\left\|f(\omega)+\left(f\left(\omega_{\alpha}\right)-f(\omega)\right)\right\|^{p}+2^{-n+1} \\
& \leq\|f(\omega)\|^{p}+2^{-n}+2^{-n+1} \\
& =\|f(\omega)\|^{p}+3 \cdot 2^{-n}
\end{aligned}
$$

and since $X$ is locally PL-convex we also have

$$
\int_{0}^{2 \pi}\left\|f(\omega)+e^{i \theta} h(\omega)\right\|^{p} \frac{d \theta}{2 \pi} \geq\|f(\omega)\|^{p} .
$$


Since $n \in \mathbb{N}$ was arbitrary, we therefore have

$$
\int_{0}^{2 \pi}\left\|f(\omega)+e^{i \theta} h(\omega)\right\|^{p} \frac{d \theta}{2 \pi}=\|f(\omega)\|^{p}
$$

for all $\omega \in \Omega$. Hence

$$
\begin{aligned}
\int_{0}^{2 \pi}\left\|f+e^{i \theta} h\right\|_{p}^{p} \frac{d \theta}{2 \pi} & =\int_{0}^{2 \pi} \int_{\Omega}\left\|f(\omega)+e^{i \theta} h(\omega)\right\|^{p} d \mu(\omega) \frac{d \theta}{2 \pi} \\
& =\int_{\Omega} \int_{0}^{2 \pi}\left\|f(\omega)+e^{i \theta} h(\omega)\right\|^{p} \frac{d \theta}{2 \pi} d \mu(\omega) \\
& =\int_{\Omega}\|f(\omega)\|^{p} d \mu(\omega) \\
& =\|f\|_{p}^{p} .
\end{aligned}
$$

Thus since $h \neq 0$ in $L^{p}(\mu, X), f$ is not a complex extreme point of $B_{L^{p}(\mu, X)}$.

Remark. Theorem 8 (b) does not hold if the restriction of $X$ being separable is removed [8]. Theorem 8 immediately gives the following improvement of $[2$, Theorem $2.5]$ :

Corollary 9. Let $X$ be a locally PL-convex continuous quasi-Banach space and let $0<p<\infty$. Then $X$ is complex strictly convex if and only if $L^{p}(\mu, X)$ is complex strictly convex.

\section{REFERENCES}

1. W.J. Davis, D.J.H. Garling and N. Tomczak-Jaegermann, The complex convexity of quasinormed spaces, J. Funct. Anal. 55 (1984), 110-150. MR 86b:46032

2. S.J. Dilworth, Complex convexity and the geometry of Banach spaces, Math. Proc. Camb. Phil. Soc. 99 (1986), 495-506. MR 87k:46032

3. P.N. Dowling, Z. Hu and M.A. Smith, Geometry of spaces of vector-valued harmonic functions, Can. J. Math. 46(2) (1994), 274-283. MR 95b:46054

4. P. Greim, An extremal vector-valued $L^{p}$-function taking no extremal vectors as values, Proc. Amer. Math. Soc. 84 (1982), 65-68. MR 83c:46034

5. P. Greim, A note on strong extreme and strongly exposed points in Bochner $L^{p}$-spaces, Proc. Amer. Math. Soc. 93 (1985), 65-66. MR 86g:46050

6. Z. Hu and D.Mupasiri, Complex strongly extreme points in quasi-normed spaces, preprint (1994).

7. J.A. Johnson, Extreme measurable selections, Proc. Amer. Math. Soc. 44 (1974), 107-112. MR 49:5818

8. D. Mupasiri, Some results on complex convexity and the geometry of complex vector spaces, Dissertation, Northern Illinois University (1992).

9. M.A. Smith, Strongly extreme points in $L^{p}(\mu, X)$, Rocky Mountain J. Math. 16 (1986), 1-5. MR 87d:46043

10. M.A. Smith, Rotundity and extremity in $\ell^{p}\left(X_{i}\right)$ and $L^{p}(\mu, X)$, Contemporary Math. 52 (1986), 143-162. MR 87h:46053

11. M.A. Smith and B. Turett, Rotundity in Lebesgue-Bochner function spaces, Trans. Amer. Math. Soc. 257 (1980), 105-118. MR 80m:46031

12. K. Sundaresan, Extreme points of the unit cell in Lebesgue-Bochner function spaces. I, Proc. Amer. Math. Soc. 23 (1969), 179-184. MR 40:719 
13. K. Sundaresan, Extreme points of the unit cell in Lebesgue-Bochner function spaces, Colloq. Math. 22 (1970), 111-119. MR 43:2493

14. E. Thorp and R. Whitley, The strong maximum modulus theorem for analytic functions into a Banach space, Proc. Amer. Math. Soc. 18 (1967), 640-646. MR 35:5643

15. Q. Xu, Inégalités pour les martingales de Hardy et renormage des espaces quasi-normés, C.R. Acad. Sci. Paris, Sér. I 306 (1988), 601-604. MR 89k:46029

16. Q. Xu, Convexités uniformes et inégalités de martingales, Math. Ann. 287 (1990), 193-211. MR 91m:46027

Department of Mathematics and Statistics, Miami University, Oxford, Ohio 45056

E-mail address: pndowling@miavx1.acs.muohio.edu

Department of Mathematics and Statistics, Miami University, Oxford, Ohio 45056

Current address: Department of Mathematics, El Paso Community College, P.O. Box 20500,

El Paso, Texas 79998

E-mail address: davidhu@laguna.epcc.edu

Department of Mathematics, University of Northern Iowa, Cedar Falls, Iowa 50614

E-mail address: mupasiri@math.uni.edu 